\title{
Optimal controller design based on minimum principle
}

\author{
Huang Da*, Huang ShuCai \\ Air Force Engineering University, 710051 shanxi xi'an, China
}

\begin{abstract}
Optimal control theory is the foundation of the modern control theory, the minimum principle in optimal control theory has a very important position, using the minimum principle to design an adaptive controller, the controller integration advantages of the principle of minimum is not affected by the control system of linear or nonlinear constraints, and the end state and free time, is accused of quantity can be controlled and are free to wait for a characteristic, using the minimum controller application example and simulation, the results show that the minimum principle of the designed controller has the ideal control effect.
\end{abstract}

\section{Introduction}

The optimal control theory is a modern control theory. Based on the frequency domain or time domain mathematical model established by the controlled object, it makes the controlled object run according to a certain control law, and makes a set of performance index reach the optimal value of [1][2]. From the mathematical point of view, the optimal control theory is to solve a class of problem functional extremum with constraints [3][4]. The minimum principle is a very important part of the optimal control theory. It is not restricted by the structure of the controlled object and is applicable to both linear and nonlinear systems [5].

The controller adjusts the control quantity in real time according to the state of the controlled object, so that the control index is stabilized near the expected value [6][7]. $\mathrm{Han} \mathrm{Pu}$ nonlinear properties of single neurons in the design of a universal adaptive PID controller based on the full application of PID control, stability and other characteristics, but it is not applicable to a plurality of controlled system [8], Liu Wei will be introduced into PID control system identification technology, design a kind of adaptive robust PID controller [9], Wang Jin fuzzy algorithm combined with PID control, the PID adaptive parameter adjustment, effective fuzzy fast, accurate, stable and intelligent PID binding characteristics of [10]. In this paper, an adaptive controller is designed based on the principle of minimum value, was charged in the model and the reference model is known, the objective function is established and the minimum controller controlled the inputs of the model, the objective function is minimized, thus the controlled variable stability in the expectation near.

\section{Controller design}

The design of controller can be divided into 4 steps: (1) the reference model is determined according to the actual system; (2) a reliable model is established for the actual system; (3) the objective function is established according to the controlled quantity; (4) the objective function is solved to get the optimal control. The design flow chart is as follows:

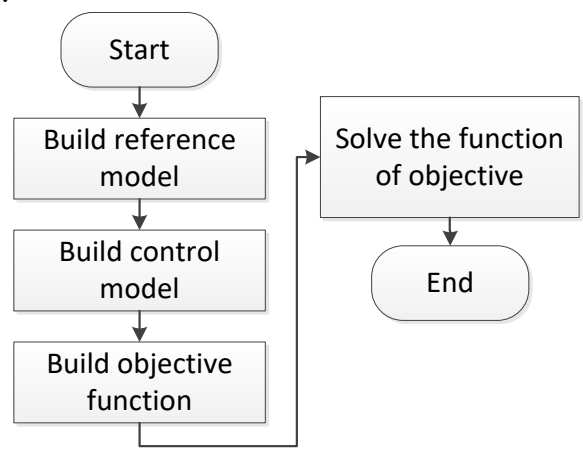

Fig.1 controller design flowchart

\subsection{The background of application of optimal controller}

The controller application system includes 3 parts: the reference model, the controlled model and the controller. The input of the controller is the objective function, and the output is the control volume. The structure diagram of the controller application system is as follows:

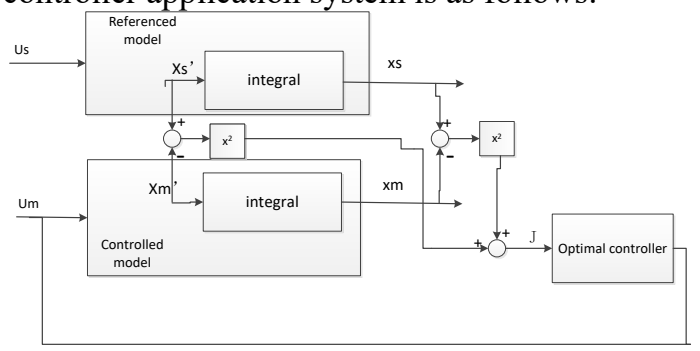

Fig.2 structure diagram of controller application system 


\subsection{Minimum principle control algorithm}

Assuming that the reference model and the controlled model have been determined, the differential mathematical expressions are as follows:

$$
\begin{gathered}
\dot{x}_{s}=f\left(x_{s}, u_{s}, t\right) \\
\dot{x}_{m}=f\left(x_{m}, u_{m}, t\right)
\end{gathered}
$$

Where Eq. (1) represents a group of differential equations of reference model, Eq. (2) represents the differential equations of the controlled model, where $\mathrm{x}$ represents the state variable, $u$ represents the system input, and $t$ represents the time. Now we need to track the state of reference state of the controlled object and set up the corresponding objective function according to this requirement. From the following two aspects of the state difference and the change of the state difference, the objective function is as follows:

$$
J=\int_{t_{0}}^{t_{f}}\left(x_{s}-x_{m}\right)^{2}+\left(\dot{x}_{s}-\dot{x}_{m}\right)^{2}+u_{m}^{2} d t
$$

According to the necessary condition of the optimal solution of the minimum principle, we can see that the optimal control solution satisfies the following series of equations [11] [12]:

(1) construct Hamiltonian function:

$$
H(x, \lambda, u)=L(x, u)+\lambda^{T}(t) f(x, u)
$$

(2) the regular equation:

$$
\dot{x}=\frac{\partial H}{\partial \lambda}, \quad \dot{\lambda}=-\frac{\partial H}{\partial x}
$$

(3) boundary conditions:

$$
x\left(t_{0}\right)=x_{0}, \lambda\left(t_{f}\right)=0
$$

(4) the absolute minimum of the Hamilton function is obtained by the relative optimal control:

$$
H\left[x^{*}(t), \lambda(t), u^{*}(t)\right]=\min H\left[x^{*}(t), \lambda(t), u(t)\right]
$$

(5) the Hamilton function at the end of the optimal trajectory should be satisfied:

$$
H\left[x^{*}(t), \lambda(t), u^{*}(t)\right]=\left\{\begin{array}{ccc}
H\left[x^{*}\left(t_{f}\right), \lambda\left(t_{f}\right), u^{*}\left(t_{f}\right)\right]=\text { const }, \text { fixed } & t_{f} \\
0, & \text { free } & t_{f}
\end{array}\right.
$$

\section{The practical application of optimal controller}

It is assumed that both the reference model and the controlled model are constant linear systems, and the mathematical expressions of the system are as follows:

$$
\begin{gathered}
\dot{x}_{s}=A_{s} x_{s}+b_{s} u_{s} \\
\dot{x}_{m}=A_{m} x_{m}+b_{m} u_{m}
\end{gathered}
$$

Where ${ }^{A_{s}}$ and ${ }^{A_{m}}$ are the system matrices of the reference model and the controlled model, and the upper formula can be synthesized as follows:

$$
\begin{gathered}
\dot{x}=\left[\begin{array}{l}
\dot{x}_{s} \\
\dot{x}_{m}
\end{array}\right]=\left[\begin{array}{cc}
A_{s} & 0 \\
0 & A_{m}
\end{array}\right]\left[\begin{array}{l}
x_{s} \\
x_{m}
\end{array}\right]+\left[\begin{array}{cc}
b_{s} & 0 \\
0 & b_{m}
\end{array}\right]\left[\begin{array}{l}
u_{s} \\
u_{m}
\end{array}\right] \\
\text { Where } \quad A=\left[\begin{array}{cc}
A_{s} & 0 \\
0 & A_{m}
\end{array}\right], B=\left[\begin{array}{cc}
b_{s} & 0 \\
0 & b_{m}
\end{array}\right], \\
x=\left[\begin{array}{l}
x_{s 1} \\
x_{s 2} \\
x_{m 1} \\
x_{m 2}
\end{array}\right], u=\left[\begin{array}{l}
u_{s} \\
u_{m}
\end{array}\right]
\end{gathered}
$$

And Eq. (11) can be expressed as:

$$
\dot{x}=A x+B u=f(x, u)
$$

Suppose:

$$
\begin{gathered}
A_{s}=\left[\begin{array}{ll}
1 & 0 \\
0 & 1
\end{array}\right], \quad b_{s}=\left[\begin{array}{l}
0 \\
1
\end{array}\right], \\
u_{s}(\mathrm{t})=\left\{\begin{array}{cc}
0 & 0 \leq t<5 \\
0.5 & 5 \leq t<10 \\
1 & 10 \leq t
\end{array}\right.
\end{gathered}
$$$$
A_{m}=\left[\begin{array}{ll}
1 & 0 \\
0 & 1
\end{array}\right], \quad b_{m}=\left[\begin{array}{l}
0 \\
1
\end{array}\right]
$$

Then:

$$
\begin{gathered}
A=\left[\begin{array}{llll}
1 & 0 & 0 & 0 \\
0 & 1 & 0 & 0 \\
0 & 0 & 1 & 0 \\
0 & 0 & 0 & 1
\end{array}\right] \\
B=\left[\begin{array}{ll}
0 & 0 \\
1 & 0 \\
0 & 0 \\
0 & 1
\end{array}\right]
\end{gathered}
$$

According to the minimum principle, the optimal control is solved. The steps of the solution are as follows:

(1) Hamilton function:

$$
\begin{aligned}
& H(x, \lambda, u) \\
& =\left(x_{s}-x_{m}\right)^{2}+\left(\dot{x}_{s}-\dot{x}_{m}\right)^{2}+u_{m}^{2}+\lambda_{1}(t) \dot{x}_{s 1} \\
& +\lambda_{2}(t) \dot{x}_{s 2}+\lambda_{3}(t) \dot{x}_{m 1}+\lambda_{4}(t) \dot{x}_{m 2} \\
& =\left(x_{s 1}-x_{m 1}\right)^{2}+\left(x_{s 2}-x_{m 2}\right)^{2}+\left(\dot{x}_{s 1}-\dot{x}_{m 1}\right)^{2} \\
& +\left(\dot{x}_{s 2}-\dot{x}_{m 2}\right)^{2}+u_{m}^{2}+\lambda_{1}(t) x_{s 1}+\lambda_{2}(t)\left(x_{s 2}\right. \\
& \left.+u_{s}\right)+\lambda_{3}(t) x_{m 1}+\lambda_{4}(t)\left(x_{m 2}+u_{m}\right)
\end{aligned}
$$

(2) regular equation: 


$$
\begin{gathered}
\left\{\begin{array} { l } 
{ \dot { x } _ { s 1 } = x _ { s 1 } } \\
{ \dot { x } _ { s 2 } = x _ { s 2 } + u _ { s } } \\
{ \dot { x } _ { m 1 } = x _ { m 1 } } \\
{ \dot { x } _ { m 2 } = x _ { m 2 } + u _ { m } }
\end{array} \Rightarrow \left\{\begin{array}{l}
x_{s 1}=a e^{t} \\
x_{s 2}=b e^{t}+u_{s} \\
x_{m 1}=c e^{t} \\
x_{m 2}=d e^{t}+u_{m}
\end{array}\right.\right. \\
\left\{\begin{array}{l}
\dot{\lambda}_{1}=2\left(x_{s 1}-x_{m 1}\right)+\lambda_{1} \\
\dot{\lambda}_{2}=2\left(x_{s 2}-x_{m 2}\right)+\lambda_{2} \\
\dot{\lambda}_{3}=-2\left(x_{s 1}-x_{m 1}\right)+\lambda_{3} \\
\dot{\lambda}_{4}=-2\left(x_{s 2}-x_{m 2}\right)+\lambda_{4}
\end{array}\right. \\
\left\{\begin{array}{l}
\dot{\lambda}_{1}=\lambda_{1}=0 \\
\dot{\lambda}_{2}=-2\left(u_{s}-u_{m}+u_{m}(0) e^{t}\right)+\lambda_{2} \\
\dot{\lambda}_{3}=\lambda_{3}=0 \\
\dot{\lambda}_{4}=2\left(u_{s}-u_{m}+u_{m}(0) e^{t}\right)+\lambda_{4}
\end{array}\right.
\end{gathered}
$$

(3) according to the boundary conditions: $\lambda\left(t_{f}\right)=0$, $x\left(t_{0}\right)=0$ we know:

$a=0, b=0, c=0, d=-u_{m}(0) \Rightarrow$

$x_{s 1}=0, x_{s 2}=u_{s}(t), x_{m 1}=0, x_{m 2}(t)=-u_{m}(0) e^{t}+u_{m}(t)$

(4)the absolute minimum of the Hamilton function is obtained by the relative optimal control:

$$
H\left[x^{*}(t), \lambda(t), u^{*}(t)\right]=\min H\left[x^{*}(t), \lambda(t), u(t)\right]
$$

Assuming that $u_{m}$ is not constrained here, the minimum value can be obtained according to the partial derivative:

$$
\frac{\partial H}{\partial u_{m}}=2 u_{m}+\lambda_{4}=0
$$

then:

$$
u_{m}^{*}(t)=-\frac{1}{2} \lambda_{4}(t)
$$

The combination of (17) and (19) forms can be obtained:

$$
\begin{aligned}
& \dot{\lambda}_{4} \\
& =2\left(u_{s}+\frac{1}{2} \lambda_{4}(t)+u_{m}(0) e^{t}\right)+\lambda_{4} \\
& =2\left(u_{s}+u_{m}(0) e^{t}\right)+2 \lambda_{4}
\end{aligned}
$$

The known system data is replaced by the upper form:

$$
\lambda_{4}(t)=\left\{\begin{array}{lc}
\frac{1}{2}\left[a_{1} e^{t}+0\right] & 0 \leq t<5 \\
\frac{1}{2}\left[b_{1} e^{t}+1\right] & 5 \leq t<10 \\
\frac{1}{2}\left[c_{1} e^{t}+2\right] & 10 \leq t \leq 15
\end{array}\right.
$$

Where ${ }^{a_{1}, b_{1}, c_{1}}$ are undetermined constant coefficients, which can be obtained by replacing the boundary conditions into the upper form.

$$
\lambda_{4}(t)=\left\{\begin{array}{cc}
0 & 0 \leq t<5 \\
e^{t-15}+1 & 5 \leq t<10 \\
-e^{t-15}+2 & 10 \leq t \leq 15
\end{array}\right.
$$

The optimal control of the type (20) is as follows:

$$
u_{m}^{*}(t)=\left\{\begin{array}{cc}
0 & 0 \leq t<5 \\
-\frac{1}{2}\left[e^{t-15}+1\right] & 5 \leq t<10 \\
-\frac{1}{2}\left[-e^{t-15}+2\right] & 10 \leq t \leq 15
\end{array}\right.
$$

\section{Simulation results}

The simulation analysis of the current constant system is carried out, the optimal system is built with simulink of MATLAB, the system structure and the simulation curve are shown as below.

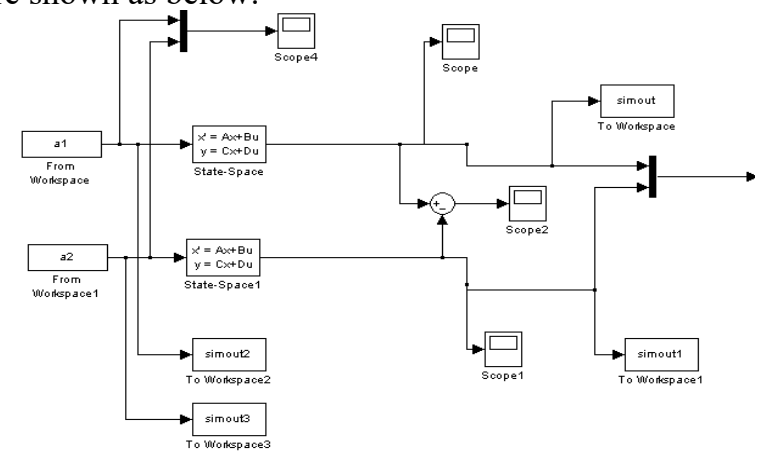

Fig. 3 structure diagram of adaptive system simulation

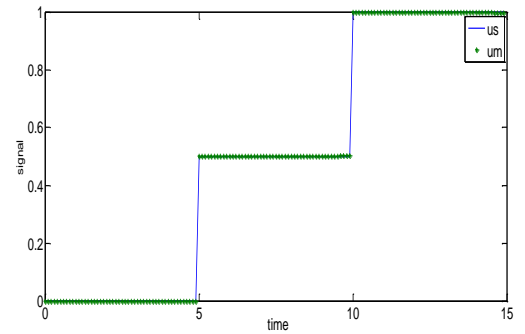

Fig. 4 reference input and controlled optimal input curve 


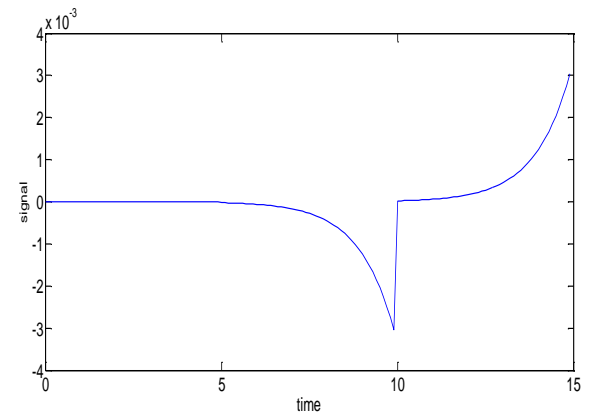

Fig. 5 input tracking error

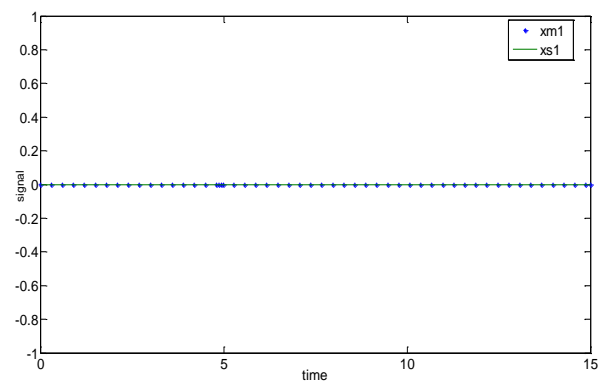

Fig. 6 status X1 tracking curve

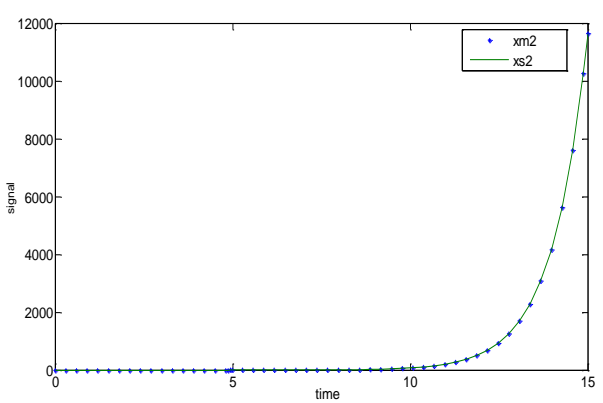

Fig.7 status $x 2$ tracking curve

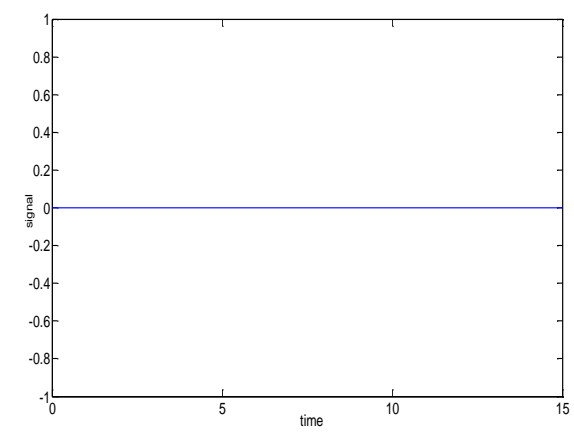

Fig. 8 state X1 tracking error curve

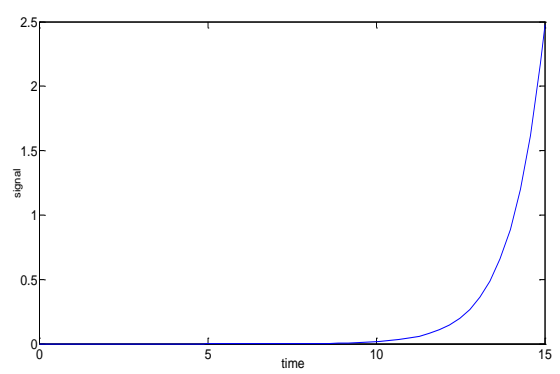

Fig. 9 state $\mathrm{x} 2$ tracking error curve

\section{Conclusion}

In instance, reference model and the controlled model should be exactly the same. in theory, calculate the optimal control input and the reference model of the input is similar to that from the above simulation results, which shows that the optimal control input and the reference input of the tracking error in the range of 0.003 , overall tracking results agree with the theoretical predictions for the target state. The function contains the simulation results of $\mathrm{X} 1$ is always 0 , tracking error free, X2 amplitude tracking error is 2.5 , compared to the state as a unit amplitude level, the tracking error in the range of 2.5. To sum up, the optimal control effect of the optimal controller designed for this example is ideal.

The optimal controller designed in this paper has (1) controlled object is not constrained by nonlinear system or linear system. (2) multiple control quantities are optimal at the same time. (3) [13] is not required for reference model structure. Generally speaking, the optimal controller of minimum principle is designed based on the objective function of the controlled quantity, and the index in the objective function can be defined according to the specific control.

\section{Reference}

1. Mahapatra P R, Shukla U S. Accurate Solution of Pure Proportional Navigation forManeuvering Targets. IEEE Transactions on Aerospace and Electronic Systems, 25(1):81-88(1989)

2. YU C J, LI B, LOXTON R, et al. Optimal discretevalued control computation[J]. Journal of Global Optimization, 56:503-518(2013)

3. KAYA C, MARTiNEZ J. Euler discretization and inexact restoration for optimal control[J].Journal of Optimization Theory and Applications,134(2):191206(2007)

4. EO $\mathrm{K} \mathrm{L}$, YANG $\mathrm{X} \mathrm{Q}$, JENNINGS $\mathrm{L} \mathrm{S}$. Computational discretization algorithms for functional inequality constrained optimization[J]. Annals of Operations Research, 98:215-234(2000)

5. G.YIN,Xian-pingGUO, Yousef TALAFHA,Nicholas A.BARAN. Near-Optimal Controls of Differential Systems with Switching and Random Jumps Subject to Fast Switching and Wideband Noise Perturbation [J]. Acta Mathematicae Applicatae Sinica. 2016

6. Dong Nuo. Nonlinear parametric fuzzy neural adaptive control of uncertain nonlinear systems [D]. Dalian Maritime University, 2014

7. Shen Yuli, Fan Bo, 1 Fuzzy - single neuron PID compound control intelligent controller [J]1 Computer Engineering, 27 (5): 92-94(2016)

8. Pu Hanjun; Wu Tongfeng; Fu Ruibin. [J] design and implementation of a universal adaptive PID controller. Journal of Guangxi University of Technology, 2009 (1): 5-9

9. Liu Wei, Zhou Junmin. Application of fuzzy adaptive PID controller in CNC machine tools [J]. mechatronics, 2010 (3): 95-96 
10. Wang Jin. Computer aided design and simulation analysis of building environment control system based on [D]. MATLAB/Simulink Tianjin University

11. Saroj K. Biswas,Shih-J. Chen,A. Satyanarayana. Optimal Temperature Tracking for Accelerated Cooling Processes in Hot Rolling of Steel[J],Dynamics and Control(4):327 340(1997)
12. Wang Wei,Tang Gongyou. Feedback and Feedforward Optimal Control for Offshore Jacket Platforms [J]. China Ocean Engineering. 2004 (04)

13. $\mathrm{Hu}$ Shousong. Optimal control theory, $[\mathrm{M}]$. Science Press,.2005 